\title{
Polymers in disordered environments
}

\author{
V. Blavatska $2^{1}$, N. Fricke ${ }^{2}$, W. Janke $e^{2 \sqrt{3}}$ \\ ${ }^{1}$ Institute for Condensed Matter Physics of the National Academy of Sciences of Ukraine, 79011 Lviv, Ukraine \\ 2 Institut für Theoretische Physik, Universität Leipzig, Postfach 100 920, 04009 Leipzig, Germany \\ ${ }^{3}$ Centre for Theoretical Sciences (NTZ), Universität Leipzig, Postfach 100 920, 04009 Leipzig, Germany
}

Received June 18, 2014, in final form July 15, 2014

\begin{abstract}
A brief review of our recent studies aiming at a better understanding of the scaling behaviour of polymers in disordered environments is given. The main emphasis is on a simple generic model where the polymers are represented by (interacting) self-avoiding walks and the disordered environment by critical percolation clusters. The scaling behaviour of the number of conformations and their average spatial extent as a function of the number of monomers and the associated critical exponents $\gamma$ and $v$ are examined with two complementary approaches: numerical chain-growth computer simulations using the PERM algorithm and complete enumerations of all possible polymer conformations employing a recently developed very efficient exact counting method.
\end{abstract}

Key words: self-avoiding walks, percolation clusters, PERM chain-growth computer simulations, exact enumerations

PACS: 64.60.al, 07.05.Tp, 64.60.ah

\section{Introduction}

Polymers in chemical and biological physics are often characterized by a linear chemical architecture and behave as (semi-)flexible chains. Typical examples of flexible polymers are synthetic polymers with a carbon backbone, such as polyethylene, where the carbon bonds along the backbone can easily rotate against each other [1-3]. Recently, much interest has been paid to semiflexible polymers, mostly because important biopolymers such as DNA, actin filaments in eukaryotic cells and some other proteins belong to this class [4, 5]. Typically, such biopolymers are supramolecular assemblies with a relatively large monomer diameter representing many atoms. Some synthetic polymers also exhibit bending stiffness over short distances along the chain. The competition between thermal energy and the bending energy of the polymer sets a characteristic length scale, the persistence length $l_{\mathrm{p}}$, describing the crossover between two main regimes: for chain lengths much larger than $l_{\mathrm{p}}$, any polymer behaves as a flexible chain, whereas for chain lengths much smaller than the persistence length, the polymer attains the limit of a rigid rod.

The conformational properties, that is size and shape of polymer coils, play an important role in models explaining the viscous flow and other hydrodynamic properties of polymer fluids. It is well established [1-3] that under good solvent conditions the conformation of a flexible chain can be characterized by a number of universal properties. This enables one to classify a broad variety of polymers, independently of their precise chemical structure, into a single universality class in space dimension $d$. Physically relevant cases are both polymers in three spatial "bulk" dimensions, $d=3$, and polymers which are completely adsorbed onto a planar substrate and hence confined to two dimensions. A simple example is the averaged radius of gyration $R_{\mathrm{G}}$, which defines the effective size of a typical $N$-monomer chain and obeys the scaling law

$$
\left\langle R_{\mathrm{G}}^{2}\right\rangle \sim N^{2 v},
$$

where the universal exponent $v$ takes on the value $v=3 / 4$ in $d=2$ dimensions [6] and $v=0.587597 \pm$ 0.000007 in $d=3$ dimensions [7] for different flexible polymers in a good solvent. Another impor- 
tant conformational characteristic is the universal averaged asphericity $A[8]$, distinguishing completely stretched, rod-like conformations $(A=1)$ from spherical ones $(A=0)$. For flexible polymers in good solvents, independent of their chemical structure, $A=0.431 \pm 0.002$ in $d=3$ [9], indicating a significant anisotropy of polymer coil conformations. Due to universality, the conformational properties of flexible macromolecules mentioned above are perfectly captured within the lattice model of self-avoiding walks (SAWs) [10].

In general, the overall size and shape of a complex polymer macromolecule are controlled by the monomer-monomer interactions, usually including van der Waals attraction and, if the constituents are charged, also long-range Coulomb interaction. In continuum models, the hard-core repulsion and van der Waals attraction can be modelled by Lennard-Jones potentials. Employing a lattice discretization of space, one considers the so-called interacting self-avoiding walks (ISAWs) (sometimes also denoted as "self-attracting self-avoiding walks (SASAWs)"), where apart from the self-avoidance constraint, representing the hard-core potential, the van der Waals contribution is modelled by a nearest-neighbour interaction,

$$
H=-\epsilon N_{i} .
$$

Here the parameter $\epsilon$ sets the energy scale and $N_{i}$ counts the number of contacts of the polymer with itself. This may be depicted as a step-like potential which qualitatively corresponds to a Lennard-Jones interaction with large-distance cutoff. Such polymer models have been applied to a large variety of problems including protein folding (where $\epsilon$ depends on the type of interacting residues) and surface adsorption [11-19]. They provide a coarse-grained approximation to flexible $\Theta$-polymers and are a suitable and very efficient way for studying generic properties of the collapse and freezing transitions for relatively long polymer chains.

In real physical processes, this ideal picture is further complicated by the fact that structural obstacles (impurities) in the environment may alter the behaviour of the system. The density fluctuations of obstacles may lead to a large spatial inhomogeneity and create pore spaces, which are frequently of fractal structure [20]. The understanding of the behaviour of macromolecules in porous environments, that is in the presence of structural disorder, e.g., in colloidal solutions or microporous membranes [21], is of great importance in polymer physics. In particular, a related problem is relevant when studying the protein folding dynamics in a cellular environment. Biological cells can be described as a highly disordered ("crowded") environment due to the presence of a large amount of soluble and insoluble biochemical species, which can be estimated to occupy up to $40 \%$ of the total aquabased volume [22, 23]. It is known that structural obstacles strongly affect protein folding and aggregation [24]. Here, we will model the disordered environment generically by forcing the SAWs to only walk on the sites of percolation clusters at the threshold value [25, 26] where they exhibit a self-similar, fractal structure [27].

As established by de Gennes [2], the scaling properties of infinitely long flexible polymers can be evaluated by analyzing the critical behaviour of the $n$-component vector spin model in the formal $n \rightarrow 0$ limit (so-called polymer limit). In particular, the polymer size exponent $v$ as defined in the scaling law (1.1) is related by universality to the correlation length critical index of the $n=0$-component model, whereas the universal shape parameters of polymer chains can be computed by field theoretic methods in terms of the critical amplitude ratios of this model [8]. Correspondingly, the problem of polymers in disordered environments can be mapped to analyses of the critical behaviour of the randomly diluted $n$-vector model in the polymer limit [28, 29].

Another class of problems concerns the behaviour of polymers in the presence of a planar substrate, which can be either energetically neutral (presenting a purely geometrical constraint), or attractive for the polymer [15-17, 30-36]. In the latter case, the polymer will be adsorbed to the surface at low temperatures and desorbed at high temperatures. The adsorption of polymers on surfaces is a long-standing problem which plays an important role in a number of technological applications (lubricants, stabilization of colloids) and biology (adsorption of biopolymers onto membranes) [37]. For example, much interest has been paid recently to experimental studies of the adsorption of peptides onto semiconductor substrates [38, 39], being of particular importance for future sensory devices and pattern recognition at the nanometer scale.

A subject of particular interest is the adsorption of polymers onto disordered (fractal) surfaces, since naturally occurring substrates are usually rough and energetically (or structurally) inhomogeneous 40 , 
41], and the fractal surface geometry is known to have a crucial effect on adsorption phenomena 42, 43]. Already simple physical arguments suggest that by increasing the surface irregularity, the number of polymer-surface contacts is strongly altered relative to the idealized planar surface without being balanced by a loss in configurational entropy. This is a consequence of changes in the probability of polymer-surface contacts with increasing roughness. A fractal surface is in general characterized by its fractal dimension $d_{\mathrm{s}}\left(d_{\mathrm{s}}=2\right.$ corresponds to a flat surface). In particular, it was shown that adsorption is enhanced (diminished), when the fractal dimension of the surface is larger (smaller) than 2 [44].

The rest of this mini-review is organized as follows. In the next section, the employed methods, PERM chain-growth computer simulations and exact enumerations, are described and their merits and drawbacks compared. Section 3 contains an overview of our results for the scaling behaviour of SAWs on "bulk" percolation clusters and a shorter paragraph on the adsorption properties to a fractal surface. Finally, in section 4 we finish with some concluding remarks and give an outlook to ongoing and future work.

\section{Methods}

Whereas for continuum polymer models Markov chain Monte Carlo (MC) and Molecular Dynamics (MD) simulations are the methods of choice [45-47], for lattice polymer models alternative numerical techniques may be employed that are better tailored to the problem at hand. The methods in a first class, chain-growth algorithms, are based on stochastic sampling, similarly to standard MC methods. In certain situations, some variants are also suitable for continuum studies [48-50]. The second type, complete or exact enumerations, on the other hand, explicitly exploit that the configuration space of lattice polymers is discrete. Complete enumerations yield exact averages of the polymer statistics for a given lattice structure. The numerical complexity of the method, however, grows in general exponentially with the number of monomers so that only relatively short chains (with of the order of $30 \div 50$ monomers) can be investigated by this means. In the next two subsections, first the specific chain-growth algorithm "PERM" will be briefly explained. Subsequently, for the special case of critical percolation clusters at the percolation threshold as depicted in figure 1 it is shown how the exponential complexity of complete enumerations can be reduced to a polynomial one when their self-similar fractal structure is exploited in a suitable way.

\subsection{PERM chain-growth algorithm}

The algorithm underlying the pruned-enriched Rosenbluth method (PERM) [51] is based on ideas from the very first days of Monte Carlo simulations, the Rosenbluth-Rosenbluth (RR) method [52] combined with enrichment strategies [53]. To grow a polymer chain, one places the $n$th monomer at a randomly chosen nearest-neighbour site of the last placed $(n-1)$ th monomer and continue iteratively until the total length of the chain $n=N$ is reached.

In order to obtain correct statistics, any attempt to place a new monomer at an already occupied site results in discarding the entire chain. This leads to the problem of an exponential "attrition", which means that the number of discarded chains grows exponentially with the chain length $N$. Clearly, this renders the method useless for long chains. In the RR method, occupied neighbours are avoided without discarding the chain. This would introduce a bias in the proper statistics which, however, can be corrected by giving a weight $W_{n} \sim \prod_{l=2}^{n} m_{l}$ to each sample conformation in the $n$th step, where $m_{l}$ is the number of free lattice sites to place the $l$ th monomer. When an ensemble of chains of total length $N$ is constructed, any new chain has to start from the same starting point, until the desired number of chain conformations is obtained. The configurational averaging for, e.g., the end-to-end distance $r \equiv \sqrt{R^{2}(N)}$ is then given by

$$
\langle r\rangle=\frac{\sum_{\text {conf }} W_{N}^{\text {conf }} r^{\text {conf }}}{\sum_{\text {conf }} W_{N}^{\text {conf }}}=\sum_{r} r P(r, N),
$$

where $W_{N}^{\text {conf }}$ is the Rosenbluth weight of an $N$-monomer chain in a given conformation and $P(r, N)$ is the distribution function for the end-to-end distance. 


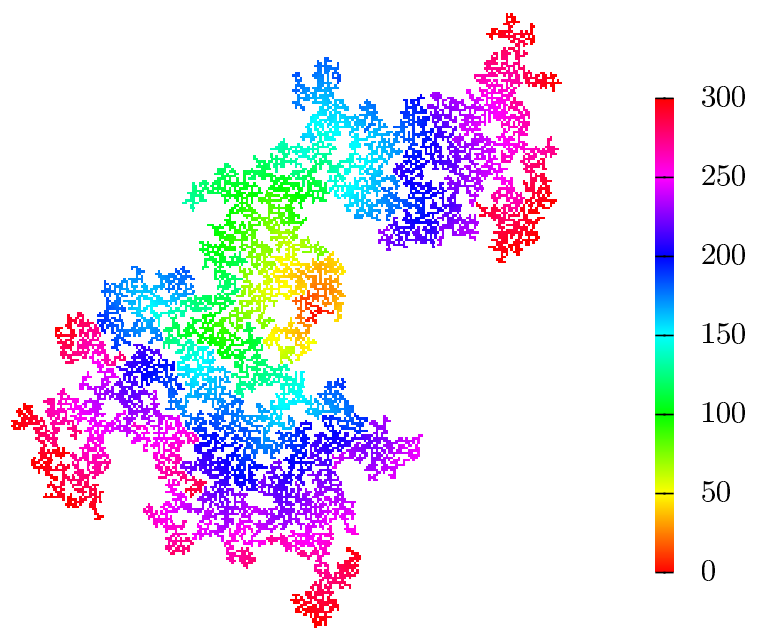

Figure 1. (Color online) Example of a critical percolation cluster in two dimensions. Colors show the chemical distance to the origin, which is in the red center. Sites more than 300 steps away are not shown.

While the chain grows by adding monomers, its Rosenbluth weight will fluctuate. PERM suppresses these fluctuations by a population control, namely by "pruning” conformations with too small weights and by "enriching" the sample with copies of high-weight conformations [51]. These copies are made while the chain is growing and continue to grow independently of each other. Pruning and enrichment are performed by choosing thresholds $W_{n}^{<}$and $W_{n}^{>}$depending on the estimate of the partition sum $Z_{n}=\sum_{\text {conf }} W_{n}^{\text {conf }}$ of the $n$-monomer chain. These thresholds are continuously updated as the simulation progresses. The zeroth iteration is a pure chain-growth algorithm without reweighting. After the first chain of full length has been obtained, we switch to $W_{n}^{<}, W_{n}^{>}$. If the current weight $W_{n}$ of an $n$-monomer chain is less than $W_{n}^{<}$, a random number $r=0,1$ is chosen; if $r=0$, the chain is discarded, otherwise it is kept and its weight is doubled. Thus, low-weight chains are pruned with probability $1 / 2$. If $W_{n}$ exceeds $W_{n}^{>}$, the conformation is doubled and the weight of each copy is taken as half the original weight. To update the threshold values, one may apply similar empirical rules as in [12, 13, 54]: $W_{n}^{>}=C\left(Z_{n} / Z_{1}\right)\left(c_{n} / c_{1}\right)^{2}$ and $W_{n}^{<}=0.2 W_{n}^{>}$, where $c_{n}$ denotes the number of the created chains having length $n$, and the parameter $C \leqslant 1$ controls the pruning-enrichment statistics. After a certain number of chains of total length $N$ is produced, the iteration is finished and a new so-called tour starts.

\subsection{Exact enumeration}

The straightforward exact enumeration method for SAWs is to use brute force and generate all possible conformations; see [55-57]. However, the computational work load becomes rapidly prohibitively high since the number of walks $Z$ increases exponentially with the number of steps $N$,

$$
Z \simeq \mu^{N} N^{\gamma-1}=N^{\gamma-1} \mathrm{e}^{N \ln \mu}
$$

Here, $\gamma$ is another universal exponent depending only on the dimension and $\mu$ is the non-universal socalled connectivity constant which besides the dimension does also depend on the details of the considered lattice.

Fortunately though, we recently discovered that the fractal structure of percolation clusters offers a way to circumvent this problem [58, 59]. The actual implementation of our method is rather complicated and will not be explained here in detail, but the basic ideas are fairly simple. The key lies in the observation that the critical clusters are very weakly connected, so that they could be divided by cutting only a small number of bonds. Thanks to the self-similarity, this applies on all length scales. We can, therefore, partition the cluster into a hierarchy of nested "blobs" with very few interconnections in order to factorize the enumeration (see figure 2). This factorization property is the main clue of the method. 


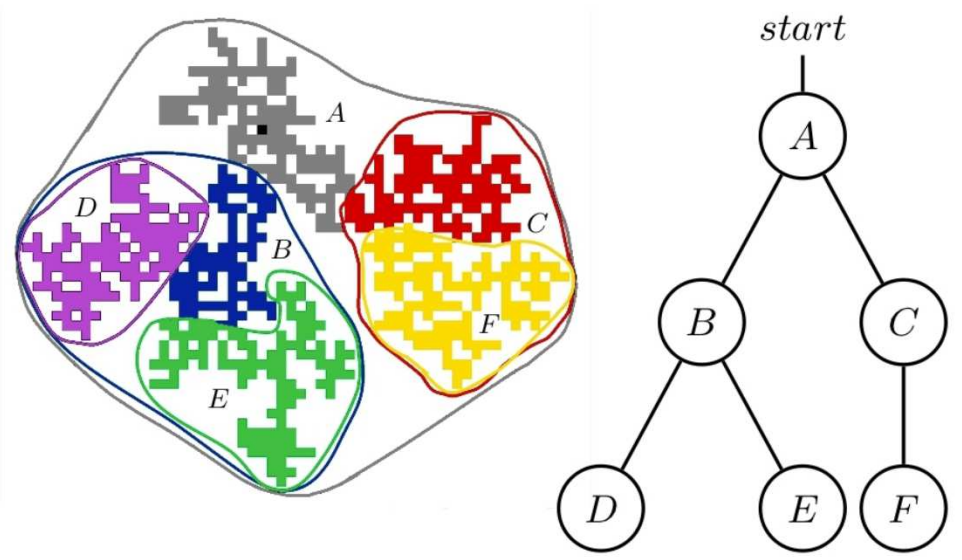

Figure 2. (Color online) Decomposition of a critical percolation cluster into nested blobs and the corresponding tree hierarchy. The starting position of the SAW is marked black (in blob $A$ ).

We start by enumerating all SAW segments of any length through the smallest blobs and divide them into different classes, depending on how they connect the different entries to the blob. For instance, if a blob has two connections ( $a$ and $b$ ) to its parent in the hierarchy, we distinguish between the following classes: segments starting at $a$ and terminating within the blob, segments starting at $b$ and terminating within the blob, and segments connecting $a$ and $b$. For the terminating segment classes, we also measure the mean distance of the chain end to the origin. We then "renormalize" the smallest blobs, treating them essentially as point-like, while we repeat the procedure for the next larger blobs that contain them. The new segments should then be matched with the right segment classes from before to determine their multiplicity and average end-point distances. This scheme is applied repeatedly going to ever larger blobs and ultimately the whole percolation cluster. Correctly implemented, this method achieves polynomial (rather that exponential) increase of computation time with the number of steps, thus allowing for walks of several thousand steps. In fact, we can exactly enumerate as many as $10^{1000}$ chain conformations which with standard techniques would take very long indeed (well, with current computers much longer than the estimated age of our Universe ...). Our recursive algorithm can be used in any dimension as is illustrated in figure 3 for the three-dimensional case, but we have only recently generalized our computer implementation to more than two dimensions [60, 61], so that results for physical quantities on percolation clusters in three or more dimensions are still somewhat premature.

The efficiency of our recursive enumeration method depends on the self-similar, fractal blob-like structure of the critical percolation clusters, allowing a factorization of the counting problem. Away from the percolation threshold, its performance deteriorates and PERM chain-growth simulations may be superior. Also, for measuring more detailed shape characteristics, such as the full gyration tensor and related universal shape invariants [62,63] as well as for a generalization to $\Theta$-polymers [63, 64], PERM is more flexible. So in this sense the two methods are complementary to each other.

\section{Results}

Let us start with our results for the scaling properties of SAWs on $d$-dimensional critical percolation clusters [65-69]. To be precise, in the PERM simulations we rather considered the (geometric) backbone of the cluster which is the subset of the cluster consisting of all bonds (or sites) through which a "current" can flow, i.e., it is the structure left when all "dangling ends" are eliminated from the cluster. The usual argument is that SAWs can be trapped in such "dangling ends", so that infinitely long chains can only exist on the backbone of the cluster.

In the PERM simulations, we adjusted the pruning-enrichment control parameter such that on average 10 chains of total length $N(=90,80,70$ in $d=2,3,4)$ are generated per iteration [12, 13], and per- 


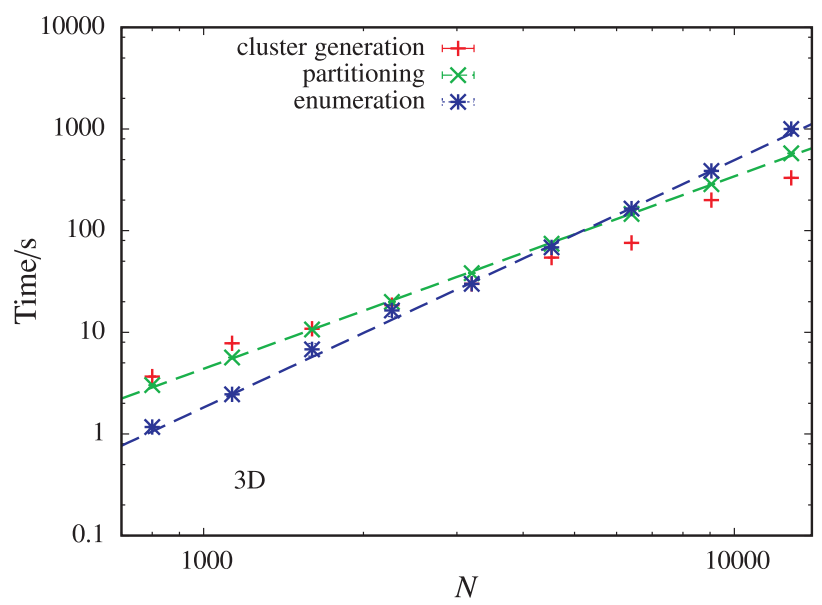

Figure 3. (Color online) Computation time (in seconds on a typical $3 \mathrm{GHz}$ PC) needed for the complete enumeration of all conformations of an $N$-step self-avoiding walk on a critical percolation cluster in three dimensions using our recently developed recursive exact enumeration algorithm. The straight lines in the log-log plot clearly indicate a power-law dependence on $N$ instead of the exponential increase for standard enumeration techniques.

formed $10^{6}$ iterations. Also, what is even more important for efficiency, we made sure that in almost all iterations at least one such a chain was created. By comparison with our exact enumeration results for randomly selected clusters, we have recently checked [70] that once PERM has managed to squeeze the chains through the bottlenecks of the percolation cluster, the convergence to the exact result is very fast. Initially, however, PERM needs a sufficient period of time to explore the landscape and to adjust the thresholds of the population control.

In the given problem we have to perform two types of averaging: The first average is performed over all SAW conformations created with PERM on a single backbone according to (2.1), and the second average is carried out over different realizations of disorder,

$$
\overline{\langle r\rangle}=\frac{1}{N_{\mathrm{c}}} \sum_{i=1}^{N_{\mathrm{c}}}\langle r\rangle_{i},
$$

where $N_{\mathrm{c}}$ is the number of different clusters. In our PERM simulations we took $N_{\mathrm{c}}=1000$.

In this way we arrived at the data for the end-to-end distance shown in figure 44 65]. To estimate the critical exponent $v_{p_{\mathrm{c}}}$, we employed in a log-log representation linear least-square fits with varying lower cutoff for the number of steps $N_{\min }$. The $\chi^{2}$ value (sum of squares of normalized deviation from the regression line) serves as a test of the goodness of fit and thus determines fit ranges where the leading scaling ansatz [1.1) is acceptable. The resulting estimates of the exponent $v_{p_{\mathrm{c}}}$ [65] are compiled in the second last line of table 1 where previously obtained results [71-76] are listed also for comparison.

With our new exact enumeration scheme, we have so far only analyzed the two-dimensional case [58, 59]. Our result for $v_{p_{\mathrm{c}}}$ in the last line of table 1 is based on walks with up to $N=1000$ steps averaged over 200000 percolation clusters at $p_{\mathrm{c}}$ (working here with the full cluster, not the backbone). In the leastsquare fit to the scaling law (1.1), the first 500 steps were excluded to reduce finite-size effects. In the meantime we have extended our exact enumeration program to up to seven dimensions, and by several technical refinements of the actual computer implementation we can now handle walks with over 10000 steps exactly. The data analysis, however, is still in progress and will be reported elsewhere [60, 61].

Estimates of the exponent $\gamma$ in (2.2) are much more difficult to obtain since $\gamma$ appears in the subleading term, which in the present context is a severe problem because $Z$ exhibits strong fluctuations from cluster to cluster. In fact, the distribution of $Z$ has log-normal character due to the fact that $Z$ is effectively a product of random variables. Still, performing least-square fits to

$$
\frac{\ln \bar{Z}}{N}=\frac{\ln A}{N}+\ln \mu+(\gamma-1) \frac{\ln N}{N},
$$




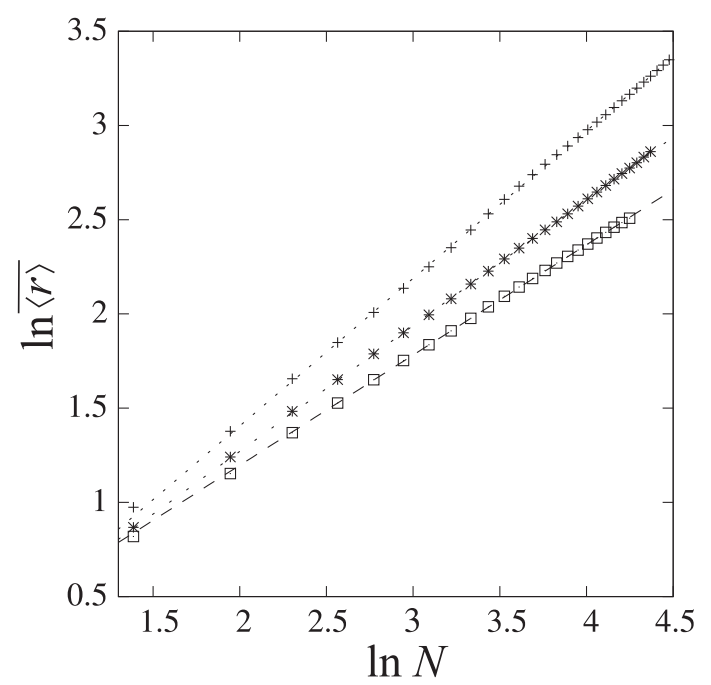

Figure 4. Disorder averaged end-to-end distance vs the number of steps obtained in PERM chain-growth simulations in double logarithmic scale for SAWs on the backbone of percolation clusters in $d=2$ (pluses), $d=3$ (stars), $d=4$ (open squares). Lines represent linear fitting, statistical error bars are of the size of symbols.

we obtain for instance in two dimensions from PERM simulations [68] the estimates $\mu=1.566 \pm 0.005$, $\gamma=1.350 \pm 0.008$ and from the exact enumerations [58] (in the interval $N=10-50$ ) $\mu=1.567 \pm 0.003$, $\gamma=1.35 \pm 0.03$, which are in surprisingly close agreement. Note that $\mu$ is basically given by the product of the connectivity constant for the regular square lattice and the percolation threshold $(2.6385 \times 0.592746=$ 1.5639) and that $\gamma$ is hardly distinguishable from its value for the regular two-dimensional lattice $(\gamma=$ $43 / 32=1.3437 \ldots$ ). With PERM we have estimated $\mu, \gamma$ also in three and four dimensions [68], whereas the exact enumeration data still have to be analyzed [60,61].

As an interesting side result, we obtained with PERM also numerical estimates for the averaged asphericity, prolateness, and size ratio of the chains in two and three dimensions [62]. All these shape characteristics can be expressed in terms of combinations of the components of the gyration tensor. They increase gradually with increasing polymer chain length - the fractal structure of the percolation cluster drives the longer polymer chain conformations to become more and more prolate. Our results quantita-

Table 1. Estimates of the exponent $v_{p_{\mathrm{c}}}$ for SAWs on critical percolation clusters. EE: exact enumerations, RG: field-theoretic renormalization group, MC: Monte Carlo simulations. For SAWs on the regular lattice one has: $v_{\mathrm{SAW}}(d=2)=3 / 4$ [6], $v_{\mathrm{SAW}}(d=3)=0.587597 \pm 0.000007$ [7], $v_{\mathrm{SAW}}(d \geqslant 4)=1 / 2$.

\begin{tabular}{|r|c|c|c|}
\hline \hline$v_{p_{\mathrm{c}}} \backslash d$ & 2 & 3 & 4 \\
\hline \hline $\mathrm{EE}[71]$ & $0.770 \pm 0.005$ & $0.660 \pm 0.005$ & \\
{$[56]$} & $0.778 \pm 0.015$ & $0.66 \pm 0.01$ & \\
{$[56]$} & $0.787 \pm 0.010$ & $0.662 \pm 0.006$ & \\
\hline $\mathrm{RG}[72]$ & 0.785 & 0.678 & 0.595 \\
{$[73]$} & 0.796 & 0.669 & 0.587 \\
\hline $\mathrm{MC}[74]$ & $0.77 \pm 0.01$ & & \\
{$[75]$} & $0.783 \pm 0.003$ & & \\
{$[76]$} & & $0.62-0.63$ & $0.56-0.57$ \\
\hline PERM [65] & $0.782 \pm 0.003$ & $0.667 \pm 0.003$ & $0.586 \pm 0.003$ \\
$\mathrm{EE}[58]$ & $0.7754 \pm 0.0015$ & & \\
\hline \hline
\end{tabular}




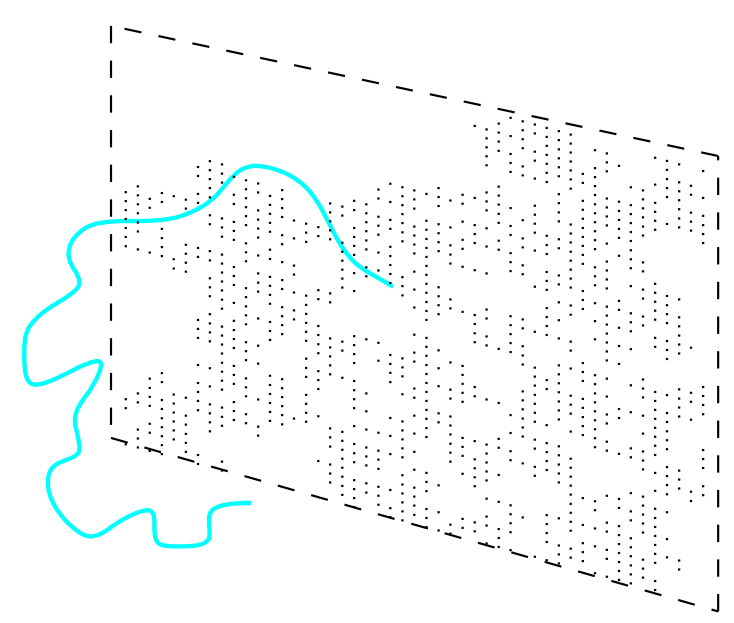

Figure 5. (Color online) Sketch of a polymer chain grafted with one end to a randomly selected site of an attractive two-dimensional percolation cluster modelling a fractal surface with fractal dimension $d_{\mathrm{s}}^{p_{\mathrm{c}}}=91 / 48 \approx 1.896<2$.

tively indicate that the shape parameters of typical polymer conformations change significantly relative to the obstacle-free case. For example, we obtained for the (universal) asphericity of SAWs in three dimensions $\left\langle A_{3}\right\rangle=0.435 \pm 0.002$ on a regular lattice in good agreement with [9] and $\overline{\left\langle A_{3}^{p_{\mathrm{c}}}\right\rangle}=0.743 \pm 0.005$ on critical percolation clusters: The shape tends to be more anisotropic and elongated due to the fractal structure of the disordered environment.

Let us finally briefly comment on the conformational statistics of polymers interacting with disordered substrates. Particularly interesting is the case of an attractive substrate, where below a critical temperature $T_{A}$, a second-order phase transition into an adsorbed state takes place. As order parameter, one considers the fraction $N_{\mathrm{s}} / N$, where $N_{\mathrm{s}}$ is the average number of monomers adsorbed to the surface and $N$ is the length of the polymer chain. In the limit of long chains, it obeys the scaling law $\left\langle N_{\mathrm{s}}\right\rangle / N \sim N^{\phi_{\mathrm{s}}-1}$, where $\phi_{\mathrm{s}}$ is the so-called surface crossover exponent [30]. In the language of lattice models, disordered surfaces can be modelled as a two-dimensional regular lattice with different types of randomly distributed defects as sketched in figure 5. Fractal properties emerge at the (two-dimensional) percolation threshold where a spanning percolation cluster of attractive sites with fractal dimension 1 $d_{\mathrm{s}}^{p_{\mathrm{c}}}=91 / 48 \approx 1.896<2$ appears [77].

In reference [78] we have studied this problem with the help of PERM. We examined the behaviour of the components of the radius of gyration $\overline{\left\langle R_{\mathrm{g} \|}^{2}\right\rangle}, \overline{\left\langle R_{\mathrm{g} \perp}^{2}\right\rangle}$ in directions parallel and perpendicular to the surface and found that the critical exponent governing the scaling of the size of the polymer chain adsorbed on the fractal substrate is larger than that for a homogeneously attractive surface. A value $v_{2}^{p_{\mathrm{c}}}=0.772 \pm 0.006$ is obtained [78, 79], to be compared with the compatible result $v_{2}^{p_{\mathrm{c}}}=0.782 \pm 0.003$ for the average size of a polymer strictly confined onto a two-dimensional percolating cluster [65, 68] and $v_{2}=0.742 \pm 0.006 \approx 3 / 4$ for a plain surface. Examining the peak structure of the heat capacity, we obtained an estimate for the surface crossover exponent of $\phi_{\mathrm{s}}^{p_{\mathrm{c}}}=0.425 \pm 0.009$, compared to $\phi_{\mathrm{s}}=0.509 \pm 0.009$ for the plain surface [78, 79]. As expected [44], the adsorption is diminished when the fractal dimension of the surface is smaller than that of the plain Euclidean surface due to the smaller number of contacts of monomers with attractive sites.

\footnotetext{
${ }^{1}$ Please note that in references $[65,66,66,68,79]$ inadvertently a wrong value of $91 / 49$ has been quoted. In table 2 of reference [68], this led to an incorrect value of $7 / 9(\approx 0.7777 \ldots)$ for a Flory-type approximation, which properly should read $144 / 187$ $(\approx 0.7700 \ldots)$.
} 


\section{Concluding remarks and outlook}

The scaling behaviour of polymers in disordered environments is a longstanding problem that has been investigated in many publications over the last 25 years. At a closer look, however, there still remain many open questions and controversial issues. In most numerical studies so far, for technical reasons the length $N$ of the polymer chains has been quite short, so that it is not guaranteed at all that the true asymptotic behaviour has been observed. This issue is for instance important when discussing whether SAWs on the backbone of a critical percolation cluster exhibit the same critical exponents as on the full cluster. Our recently further improved exact enumeration scheme [60] allows us now for the first time to study the chains in up to $d=7$ dimensions with over 10000 monomers exactly and hence offers a completely new perspective. Our preliminary data analysis indeed suggests that new insights into an old problem may be expected in the near future.

It is also possible to generalize our enumeration method to $\Theta$-polymers in disordered environments which enables us to study with unprecedented accuracy the collapse and freezing transitions of IASWs on critical percolation clusters. A stretching force [26, 57, 63, 64, 80, 81] can also be taken into account. Finally, a careful comparison with related random walk types, e.g., kinetic growth walks [82], may shed new light on the rather involved statistical properties of polymers in disordered environments.

\section{Acknowledgements}

This work has been supported by an Institute Partnership Grant "Leipzig-Lviv" of the Alexander von Humboldt Foundation. Financial support by the Sächsische DFG Forschergruppe FOR877 under Grant No. JA 483/29-1 and the DFG Sonderforschungsbereich SFB/TRR 102 (project B04) is gratefully acknowledged.

\section{References}

1. Flory P., Principles of Polymer Chemistry, Cornell University Press, Ithaca, 1953.

2. de Gennes P.-G., Scaling Concepts in Polymer Physics, Cornell University Press, Ithaca and London, 1979.

3. Des Cloizeaux J., Jannink G., Polymers in Solution, Clarendon Press, Oxford, 1990.

4. Bustamante C., Marko J.F., Siggia E.D., Smith S., Science, 1994, 265, 1599; doi 10.1126/science.8079175

5. Ober C.K., Science, 2000, 288, 448; doi $10.1126 /$ science.288.5465.448

6. Nienhuis B., Phys. Rev. Lett., 1982, 49, 1062; doi 10.1103/PhysRevLett.49.1062

7. Clisby N., Phys. Rev. Lett., 2010, 104, 055702; doi 10.1103/PhysRevLett.104.055702

8. Aronovitz J.A., Nelson D.R., J. Phys. France, 1986, 47, 1445; doi 10.1051/jphys:019860047090144500

9. Bishop M., Saltiel C.J., J. Chem. Phys., 1988, 88, 6594; doi 10.1063/1.454446

10. Vanderzande C., Lattice Models of Polymers, Cambridge University Press, Cambridge, 1998.

11. Lau K.F., Dill K.A., Macromolecules, 1989, 22, 3986; doi 10.1021/ma00200a030

12. Bachmann M., Janke W., Phys. Rev. Lett., 2003, 91, 208105; doi 10.1103/PhysRevLett.91.208105

13. Bachmann M., Janke W., J. Chem. Phys., 2004, 120, 6779; doi 10.1063/1.1651055

14. Schiemann R., Bachmann M., Janke W., J. Chem. Phys., 2005, 122, 114705; doi 10.1063/1.1814941

15. Bachmann M., Janke W., Phys. Rev. Lett., 2005, 95, 058102; doi 10.1103/PhysRevLett.95.058102

16. Bachmann M., Janke W., Phys. Rev. E, 2006, 73, 020901(R); doi 10.1103/PhysRevE.73.020901

17. Bachmann M., Janke W., Phys. Rev. E, 2006, 73, 041802; doi 10.1103/PhysRevE.73.041802

18. Bachmann M., Janke W., In: Rugged Free Energy Landscapes, Janke W. (Ed.), Lecture Notes in Physics Vol. 736, Springer, Berlin, 2008, 203-246; doi 10.1007/978-3-540-74029-2_8

19. Bachmann M., Janke W., AIP Conf. Proc., 2008, 1071, 1; doi 10.1063/1.3033357

20. Dullen A.L., Porous Media: Fluid Transport and Pore Structure, Academic, New York, 1979.

21. Cannell D.S., Rondelez F., Macromolecules, 1980, 13, 1599; doi 10.1021/ma60078a046

22. Minton A.P., J. Biol. Chem., 2001, 276, 10577; doi 10.1074/jbc.R100005200.

23. Ellis R.J., Minton A.P., Nature, 2003, 425, 27; doi 10.1038/425027a

24. Horwich A., Nature, 2004, 431, 520; doi $10.1038 / 431520$ a

25. Barat K., Chakrabarti B.K., Phys. Rep., 1995, 258, 378; doi 10.1016/0370-1573(95)00009-6

26. Kumar S., Li M.S., Phys. Rep., 2010, 486, 1; doi 10.1016/j.physrep.2009.11.001

27. Stauffer D., Aharony A., Introduction to Percolation Theory, Taylor and Francis, London, 1992. 
28. Kim Y., J. Phys. C: Solid State Phys., 1983, 16, 1345; doi 10.1088/0022-3719/16/8/005

29. Blavats'ka V., von Ferber C., Holovatch Yu., Phys. Rev. E, 2001, 64, 041102; doi 10.1103/PhysRevE.64.041102.

30. Eisenriegler E., Kremer K., Binder K., J. Chem. Phys., 1982, 77, 6296; doi 10.1063/1.443835

31. Eisenriegler E., Polymers Near Surfaces: Conformation Properties and Relation to Critical Phenomena, World Scientific, Singapore, 1993.

32. Möddel M., Bachmann M., Janke W., J. Phys. Chem. B, 2009, 113, 3314; doi 10.1021/jp808124v.

33. Möddel M., Janke W., Bachmann M., Phys. Chem. Chem. Phys., 2010, 12, 11548; doi 10.1039/c002862b

34. Möddel M., Janke W., Bachmann M., Comput. Phys. Commun., 2011, 182, 1961; doi 10.1016/j.cpc.2010.12.016

35. Möddel M., Janke W., Bachmann M., Macromolecules, 2011, 44, 9013; doi 10.1021/ma201307c

36. Möddel M., Janke W., Bachmann M., Phys. Rev. Lett., 2014, 112, 148303; doi 10.1103/PhysRevLett.112.148303

37. Xie A.F., Granick S., Nat. Mater., 2002, 1, 129; doi $10.1038 /$ nmat738

38. Whaley S.R., English D.S., Hu E.L., Barbara P.F., Belcher A.M., Nature, 2000, 405, 665; doi 10.1038/35015043

39. Bachmann M., Goede K., Beck-Sickinger A., Grundmann M., Irbäck A., Janke W., Angew. Chem. Int. Ed., 2010, 49, 9530; doi 10.1002/anie.201000984

40. Avnir D., Farin D., Pfeifer P., J. Chem. Phys., 1983, 79, 3566; doi 10.1063/1.446211

41. Avnir D., Farin D., Pfeifer P., Nature, 1984, 308, 261; doi 10.1038/308261a0

42. Kawaguchi M., Arai T., Macromolecules, 1991, 24, 889; doi 10.1021/ma00004a013

43. Huber G., Vilgis T.A., Eur. Phys. J. B, 1998, 3, 217; doi 10.1007/s100510050306

44. Bouchaud E., Vannimenus J., J. Phys. France, 1989, 50, 2931; doi 10.1051/jphys:0198900500190293100

45. Janke W., In: Ageing and the Glass Transition, Henkel M., Pleimling M., Sanctuary R. (Eds.), Lecture Notes in Physics Vol. 716, Springer, Berlin, 2007, 207-260; doi 10.1007/3-540-69684-9_5

46. Janke W., In: Computational Many-Particle Physics, Fehske H., Schneider R., Weisse A. (Eds.), Lecture Notes in Physics Vol. 739, Springer, Berlin, 2008, 79-140; doi 10.1007/978-3-540-74686-7_4

47. Janke W., In: Order, Disorder and Criticality: Advanced Problems of Phase Transition Theory, Vol. 3, Holovatch Yu. (Ed.), World Scientific, Singapore, 2012, 93-166.

48. Garel T., Orland H., J. Phys. A: Math. Gen., 1990, 23, L621; doi 10.1088/0305-4470/23/12/007

49. Schöbl S., Zierenberg J., Janke W., Phys. Rev. E, 2011, 84, 051805; doi 10.1103/PhysRevE.84.051805

50. Schöbl S., Zierenberg J., Janke W., J. Phys. A: Math. Theor., 2012, 45, 475002; doi 10.1088/1751-8113/45/47/475002

51. Grassberger P., Phys. Rev. E, 1997, 56, 3682; doi 10.1103/PhysRevE.56.3682

52. Rosenbluth M.N., Rosenbluth A.W., J. Chem. Phys., 1955, 23, 356; doi 10.1063/1.1741967

53. Wall F.T., Erpenbeck J.J., J. Chem. Phys., 1959, 30, 634; doi $10.1063 / 1.1730021$

54. Hsu H.P., Mehra V., Nadler W., Grassberger P., J. Chem. Phys., 2003, 118, 444; doi 10.1063/1.1522710.

55. Lam P.M., J. Phys. A: Math. Gen., 1990, 23, L831; doi 10.1088/0305-4470/23/16/010

56. Ordemann A., Porto M., Roman H.E., Bunde A., Phys. Rev. E, 2000, 61, 6858; doi 10.1103/PhysRevE.61.6858

57. Singh A.R., Giri D., Kumar S., Phys. Rev. E, 2009, 79, 051801; doi 10.1103/PhysRevE.79.051801

58. Fricke N., Janke W., Europhys. Lett., 2012, 99, 56005; doi 10.1209/0295-5075/99/56005

59. Fricke N., Janke W., Physics Procedia, 2012, 34, 39; doi 10.1016/j.phpro.2012.05.006

60. Fricke N., Janke W., Leipzig preprint (to be published).

61. Fricke N., Janke W., Preprint arXiv:1409.3457 2014.

62. Blavatska V., Janke W., J. Chem. Phys., 2010, 133, 184903; doi $10.1063 / 1.3501368$

63. Blavatska V., Janke W., Comput. Phys. Commun., 2011, 182, 1966; doi 10.1016/j.cpc.2010.12.022

64. Blavatska V., Janke W., Phys. Rev. E, 2009, 80, 051805; doi 10.1103/PhysRevE.80.051805

65. Blavatska V., Janke W., Europhys. Lett., 2008, 82, 66006; doi 10.1209/0295-5075/82/66006

66. Blavatska V., Janke W., Phys. Rev. Lett., 2008, 101, 125701; doi 10.1103/PhysRevLett.101.125701.

67. Blavatska V., Janke W., In: Proceedings of the International Conference "Path Integrals - New Trends and Perspectives” (Dresden, 2007), Janke W., Pelster A. (Eds.), World Scientific, Singapore, 2008, 585-588.

68. Blavatska V., Janke W., J. Phys. A: Math. Theor., 2009, 42, 015001; doi 10.1088/1751-8113/42/1/015001

69. Blavatska V., Janke W., Physics Procedia, 2010, 3, 1431; doi 10.1016/j.phpro.2010.01.202

70. Fricke N., Janke W., Eur. Phys. J. Special Topics, 2013, 216, 175; doi 10.1140/epjst/e2013-01740-4

71. Rintoul M.D., Moon J., Nakanishi H., Phys. Rev. E, 1994, 49, 2790; doi 10.1103/PhysRevE.49.2790

72. Von Ferber C., Blavatska V., Folk R., Holovatch Yu., Phys. Rev. E, 2004, 70, 035104(R); doi 10.1103/PhysRevE.70.035104

73. Janssen H.-K., Stenull O., Phys. Rev. E, 2007, 75, 020801(R); doi 10.1103/PhysRevE.75.020801

74. Woo K.Y., Lee S.B., Phys. Rev. A, 1991, 44, 999; doi 10.1103/PhysRevA.44.999

75. Grassberger P., J. Phys. A: Math. Gen., 1993, 26, 1023; doi 10.1088/0305-4470/26/5/022

76. Lee S.B., J. Korean Phys. Soc., 1996, 29, 1.

77. Havlin S., Ben Abraham D., Adv. Phys., 1987, 36, 695; doi $10.1080 / 00018738700101072$

78. Blavatska V., Janke W., J. Chem. Phys., 2012, 136, 104907; doi 10.1063/1.3691102 
79. Blavatska V., Janke W., Physics Procedia, 2012, 34, 55; doi 10.1016/j.phpro.2012.05.009

80. Kumar S., Giri D., Phys. Rev. Lett., 2007, 98, 048101; doi 10.1103/PhysRevLett.98.048101

81. Kumar S., Jensen I., Jacobsen J.L., Guttmann A.J., Phys. Rev. Lett., 2007, 98, 128101; doi 10.1103/PhysRevLett.98.128101

82. Fricke N., Bock J., Janke W., diffusion-fundamentals.org, 2013, 20, 111.

\title{
Полімери у невпорядкованих середовищах
}

\author{
В. Блавацька $2^{17}$, Н. Фріке $e^{2}$, В. Янке $e^{213}$ \\ 1 Інститут фізики конденсованих систем Національної академії наук України, 79011 Львів, Україна \\ 2 Інститут теоретичної фізики, Університет Лейпцига, 04009 Лейпциг, Німеччина \\ 3 Центр теоретичних досліджень (NTZ), Університет Лейпцига, 04009 Лейпциг, Німеччина
}

Подаємо короткий огляд наших недавніх досліджень, метою яких $є$ краще розуміння скейлінгової поведінки полімерів у невпорядкованих середовищах. Основна увага приділяється простій узагальненій моделі, в рамках якої полімери представляються як (самовзаємодіючі) випадкові блукання із самоперетинами, а невпорядковане середовище - як критичний перколяційний кластер. Проаналізовано скейлінгову поведінку кількості можливих конформацій та усередненого просторового видовження полімера як функції кількості мономерів, а також отримано значення відповідних критичних показників $\gamma$ та $v$, застосовуючи два взаємодоповнюючі підходи: чисельні симуляції в рамках алгоритму зростаючого ланцюжка (PERM) та точний перерахунок всіх можливих конформацій із використанням недавно розвиненої дуже ефективної методики.

Ключові слова: випадкові блукання, перколяційний кластер, комп'ютерні симуляції зростаючого ланцюжка (PERM), методи точного перерахунку 


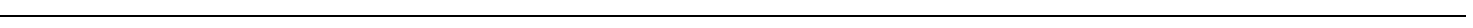

